\title{
Innovations and advances in instrumentation at the W. M. Keck Observatory
}

Marc Kassis, Dwight Chan, Shui Kwok, Tomas Krasuski, James E. Lyke, et al.

Marc Kassis, Dwight Chan, Shui Kwok, Tomas Krasuski, James E. Lyke, Sam Ragland, Scott Lilley, Sylvain Cetre, Peter Wizinowich, Hilton A. Lewis, Percy Gomez, Luca Rizzi, James E. Larkin, Tuan Do, Michael P. Fitzgerald, Andy Skemer, J. Xavier Prochaska, Kyle Westfall, Ben Mazin, Dimitri Mawet, Keith Matthews, Christopher Martin, Andrew W. Howard, Jessica R. Lu, Mark R. Chun, "Innovations and advances in instrumentation at the W. M. Keck Observatory," Proc. SPIE 10702, Ground-based and Airborne Instrumentation for Astronomy VII, 1070207 (6 July 2018); doi: 10.1117/12.2312316

Event: SPIE Astronomical Telescopes + Instrumentation, 2018, Austin, Texas, United States 


\title{
Innovations and advances in instrumentation at the W. M. Keck Observatory
}

Marc Kassis*a $^{\text {a }}$ Dwight Chan ${ }^{\mathrm{a}}$, Shui Kwok ${ }^{\mathrm{a}}$, Tomas Krasuski ${ }^{\mathrm{a}}$, James E. Lyke ${ }^{\mathrm{a}}$, Sam Ragland ${ }^{\mathrm{a}}$, Scott Lilley $^{\mathrm{a}}$, Sylvain Cetre ${ }^{\mathrm{a}}$, Peter Wizinowich ${ }^{\mathrm{a}}$, Hilton A. Lewis ${ }^{\mathrm{a}}$, Percy Gomez ${ }^{\mathrm{a}}$, Luca Rizzi ${ }^{\mathrm{a}}$, James E.

Larkin $^{\mathrm{b}}$, Tuan Do ${ }^{\mathrm{b}}$, Michael P. Fitzgerald ${ }^{\mathrm{b}}$, Andy Skemer ${ }^{\mathrm{c}}$, J. Xavier Prochaska ${ }^{\mathrm{c}}$, Kyle Westfall ${ }^{\mathrm{c}}$, Ben Mazin $^{\mathrm{d}}$, Dimitri Mawet ${ }^{\mathrm{e}}$, Keith Matthews ${ }^{\mathrm{e}}$, Christopher Martin ${ }^{\mathrm{e}}$, Andrew W. Howard ${ }^{\mathrm{e}}$, Jessica R. $\mathrm{Lu}^{\mathrm{f}}$, Mark R. Chun ${ }^{\mathrm{g}}$

${ }^{\mathrm{a}}$ W. M. Keck Observatory, 65-1120 Mamalahoa Hwy, Kamuela, HI 96743; ${ }^{b}$ University of California Los Angeles, Box 951547, Los Angeles, CA 90095-1547; ' University of California Santa Cruz, 1156 High Street, Santa Cruz, CA 95064; ${ }^{\mathrm{d}}$ University of California Santa Barbara, Santa Barbara, CA 93106; 'California Institute of Technology, 1200 E. California Blvd., Pasadena, CA 91125; f University of California Berkeley, Campbell Hall, Berkeley, CA 94720; ${ }^{\mathrm{g}}$ Institute for Astronomy, University of Hawaii, 640 N. A’ohoku Place, Hilo, HI 96720

\begin{abstract}
Since the start of operations in 1993, the twin 10 meter W. M. Keck Observatory telescopes have continued to maximize their scientific impact and to produce transformative discoveries that keep the observing community on the frontiers of astronomical research. Upgraded capabilities and new instrumentation are provided though collaborative partnerships with Caltech and UC instrument development teams. The observatory adapts and responds to the observers' evolving needs as defined in the observatory's strategic plan, periodically refreshed in collaboration with the science community. This paper summarizes the performance of recently commissioned infrastructure projects, technology upgrades, and new additions to the suite of instrumentation at the observatory. We will also provide a status of projects currently in the design or development phase, and since we need to keep our eye on the future, we mention projects in exploratory phases that originate from our strategic plan.

Recently commissioned projects include telescope control system upgrades, OSIRIS spectrometer and imager upgrades, and deployments of the Keck Cosmic Web Imager (KCWI), the Near-Infrared Echellette Spectrometer (NIRES), and the Keck I Deployable Tertiary Mirror (KIDM3). Under development are upgrades to the NIRSPEC instrument and adaptive optics (AO) system. Major instrumentation in design phases include the Keck Cosmic Reionization Mapper and the Keck Planet Finder. Future instrumentation studies and proposals underway include a Ground Layer Adaptive Optics system, NIRC2 upgrades, the energy sensitive instrument KRAKENS, an integral field spectrograph LIGER, and a laser tomography AO upgrade. Last, we briefly discuss recovering MOSFIRE and its return to science operations.
\end{abstract}

Keywords: Infrared, Instrumentation, Integral Field, Laser Guide Star, Spectrograph, Telescope Control, Adaptive Optics

\section{INTRODUCTION}

Located atop Maunakea on the island of Hawaii, the W. M. Keck Observatory (WMKO), with its twin 10-m telescopes, has a history of transformative discoveries, instrumental advances, and education for young scientists since the start of science operations in 1993. Observing time is available primarily to Caltech, University of California (UC), NASA, and University of Hawaii (UH) and some nights are also available through Yale, Notre Dame, Northwestern University, and Swinburne University as well as NOAJ through a time exchange with Subaru. To maintain the scientific leadership of this observing community, WMKO develops and maintains state-of-the-art instrumentation and systems that keep the observer's science at the cutting edge in astronomy. The observers use well-designed, work- horse instruments that, when combined with the 10-m aperture and excellent Maunakea seeing, offer high sensitivity measurements. Nightly operations focus on maximizing efficient data acquisition with an "astronomer first" approach that allows for agility and flexibility.

Ground-based and Airborne Instrumentation for Astronomy VII, edited by Christopher J. Evans, Luc Simard, Hideki Takami, Proc. of SPIE Vol. 10702, 1070207 · (c) 2018 SPIE · CCC code: 0277-786X/18/\$18 · doi: 10.1117/12.2312316 
Our observers use a current suite of ten instruments with four working on Keck I and six on Keck II. These instruments include three multi-object spectrographs that operate at optical (LRIS and DEIMOS) and near-infrared (MOSFIRE) wavelengths, high-resolution and low-resolution infrared spectrographs (NIRSPEC and NIRES), a high-resolution optical spectrograph (ESI), a seeing limited optical IFU (KCWI), an infrared IFU (OSIRIS) that operates behind an adaptive optics (AO) system, a high spatial resolution imager (NIRC2) behind an AO system, and a high-resolution precision radial velocity spectrograph (HIRES). These instruments have designated locations on the telescope where they operate that includes Cassegrain, bent Cassegrain, and Nasmyth ports.

To maximize WMKO's scientific impact, WMKO's community of instrument developers currently at Caltech, The University of California at Los Angeles, Santa Barbra, and Santa Cruz (UCLA, UCSB, UCSC), and the Space Science Laboratory (SSL) in Berkeley are developing new capabilities and upgrading existing facility instruments. The initiatives are driven by our strategic plan that was developed under the leadership of the WMKO Science Steering Committee (SSC), Directors at Palomar and Lick Observatories, as well as the WMKO Director and Chief Scientist, and was last updated in 2016. The broader WMKO astronomical community provided input by identifying synergies with other facilities and evaluating the potential of new initiatives for enabling scientific discovery. Recent developments towards achieving the goals presented in the strategic plan are described below and include two new instruments, upgrades to existing facility instruments, projects that are in development stages, and new instrument concepts under consideration.

\section{COMMISSIONED INSTRUMENTATION}

\subsection{Keck Cosmic Web Imager}

On 12 April 2017, the Keck Cosmic Web Imager ${ }^{2}$ (KCWI) achieved first light ${ }^{3}$ and started routine operations on the Keck II telescope in the 2017B observing semester with 71 nights scheduled in its first year of operation. KCWI was designed and built by a collaborative team from Caltech, UCSC, and WMKO with Dr. Christopher Martin as the principle investigator. KCWI is a wide-field integral-field seeing limited spectrograph optimized for precision sky limited spectroscopy of low surface brightness phenomena. KCWI features high throughput and flexibility in its field of view (FOV), spatial sampling, bandpass, and spectral resolution.



Figure 1. The "first-light" image from KCWI (middle) shows more than 100 stars in the core of the globular cluster Messier 3 (right). The boxes are positioned around some fo the stars and connected to their corresponding spectra. The green outlines showcase the highest spectral-resolution $(\mathrm{R} \sim 18,000)$ setting of KCWI while the other colors represent lower resolutions spanning R 900 to 9,000. Each star has a rich spectrum of absorption lines that contain information about the velocity and chemical composition of the star. 
As a facility instrument, KCWI is intended to be a two-armed spectrograph with an optimized blue and red side. The delivered instrument consists only of the blue arm, while the red arm started a preliminary design phase (3.2). The initial development of KCWI blue provides the Keck observing community with wavelength coverage from 350-560 nm. The instrument operates in a fixed gravity orientation at the Keck II right Nasmyth focal station and corrects for field rotation using a K-mirror for the science FOV. KCWI's integral field unit (IFU) uses a small, medium, and large selectable slicer mirror stacks to provide 0.35 ", 0.69 " or 1.38 " spatial resolution that results in FOVs of 20.4" x 8.4", 16.5", or 33.1" for the three configurations. There are currently four Volume Phase Holographic (VPH) gratings that provide resolutions of R 900 to 18,000 in combination with the image slicer scales. To select a wavelength of interest, the dewar rides on an articulating stage that positions the camera at an angle relative to the VPH grating. For a complete description of the instrument and its capabilities please see the instrument KCWI's on-line web documentation ${ }^{5}$.

\subsection{Near InfraRed Echellette Spectrometer}

Led by PIs Keith Matthews and Tom Soifer at Caltech, the Near-InfraRed Echellette Spectrometer (NIRES) is a prism cross dispersed near-infrared spectrometer mounted on the Keck II telescope at a Right Bent Cassegrain (RBC) port. It is equipped with a separate K-band slit-viewing camera that enables real-time source identification and that will provide guiding on slit starting in the fall. An additional optical camera common for all WMKO instruments is available for offset guiding. After being developed entirely at Caltech, NIRES was delivered to the observatory in April 2017, was integrated with the telescope during the 2017B semester, and started routine night observations in February 2018.

To prepare for the NIRES installation, a NEMA 4 electronics enclosure was installed next to RBC port \#2. The enclosure houses an EIA 19 inch rack with approximately 10 units of rack space available for instrument specific electronics. A custom glycol panel was installed at the RBC platform to manage the glycol for the guider, electronics enclosure heat exchanger, and the detector electronics mounted on the instrument. A new cable wrap was installed to accommodate the $420^{\circ}$ range of motion of the rotator. In total, close to $2000 \mathrm{lbs}$. was added to the elevation ring of the Keck II telescope. This includes over $950 \mathrm{lbs}$. of equipment and infrastructure, $870 \mathrm{lbs}$. for NIRES, and an additional 110 lbs. of liquid Nitrogen for cooling of the instrument.

NIRES has a liquid Nitrogen fill capacity of $60 \mathrm{~L}$ and this capacity would allow NIRES to remain at operating temperatures for three days without filling. However, the 20L/day of Nitrogen boil-off translates to about $36 \mathrm{lbs}$. of weight change to the telescope balance. Thus, to minimize telescope imbalances, NIRES is filled on a daily basis. Because NIRES is mounted to the elevation ring, the telescope is restricted to be at Zenith during the daily fill, and this puts a strain on telescope resources and restricting telescope access for about an hour each day.

To alleviate the daily telescope restrictions during the liquid Nitrogen fills a potential upgrade to replace the liquid Nitrogen with a CCR system is being considered. To accommodate the addition of the CCR Helium lines and power cable, the rotator cable wrap will be upgraded to a larger size. This upgrade will also include changing the cable wrap infrastructure. The design can then be changed to increase the rotator range of motion to $>500^{\circ}$. Eliminating the liquid Nitrogen fills would remove the daily telescope restrictions and free up WMKO support staff. A conceptual study for a CCR system is being completed in 2019 with the intention of upgrading NIRES in 2020.

\subsection{Telescope Control System Upgrade}

Launched in 2009, WMKO's Telescope Control System Upgrade ${ }^{5,6}$ (TCSU) project set out to improve telescope pointing, tracking, and offsetting performance as well as to increase maintainability and reliability. WMKO started operating with TCSU on Keck II on a nightly basis in October 2017. A majority of the instrumentation on Keck I also uses TCSU routinely, and we anticipate all Keck I instrumentation operating with TCSU by the end of the summer. WMKO is maintaining the ability to revert to the old system as a backup except for our two newest instruments, KCWI and NIRES, that were commissioned using TCSU on the Keck II telescope.

There were several challenges of implementing TCSU in particular because WMKO took an approach of integrating the hardware and software while never requiring a shutdown of either telescope. To mitigate the challenge of accessing the telescopes for testing and to reduce the risk of damaging instrumentation, a rotator simulator was setup in the lab. The achieved performance, in terms of pointing, tracking, maintainability and safety is a great improvement compared to the old system.

The TCSU project replaced all of the major elements of the telescope controls, rotator and secondary mirror controls, and safety system. National Instrument's reconfigurable I/O technology (i.e. RIO), with their embedded field programmable gate arrays are used as the core of the telescope's digital velocity control loop, structural filter, and 
tachometer filter. They were also used to create a monitoring and safety shutdown system for the rotator velocity controller that has uncovered previously unknown issues in the instrument and telescope positioning. Delta Tau's programmable multi-axis controllers (PMAC), are used to control the rotator and secondary mirror. The PMACs enable better tuning and faster slew speeds. An Allen Bradley's ControlLogix controller and the family of Flex IO modules were used to create a distributed safety system able to handle a wide variety of signal types. Modern Linux based computers replaced VxWorks stations for control. This technology refresh based on commercial off the shelf equipment will allow us to get rid of our obsolete and custom equipment.

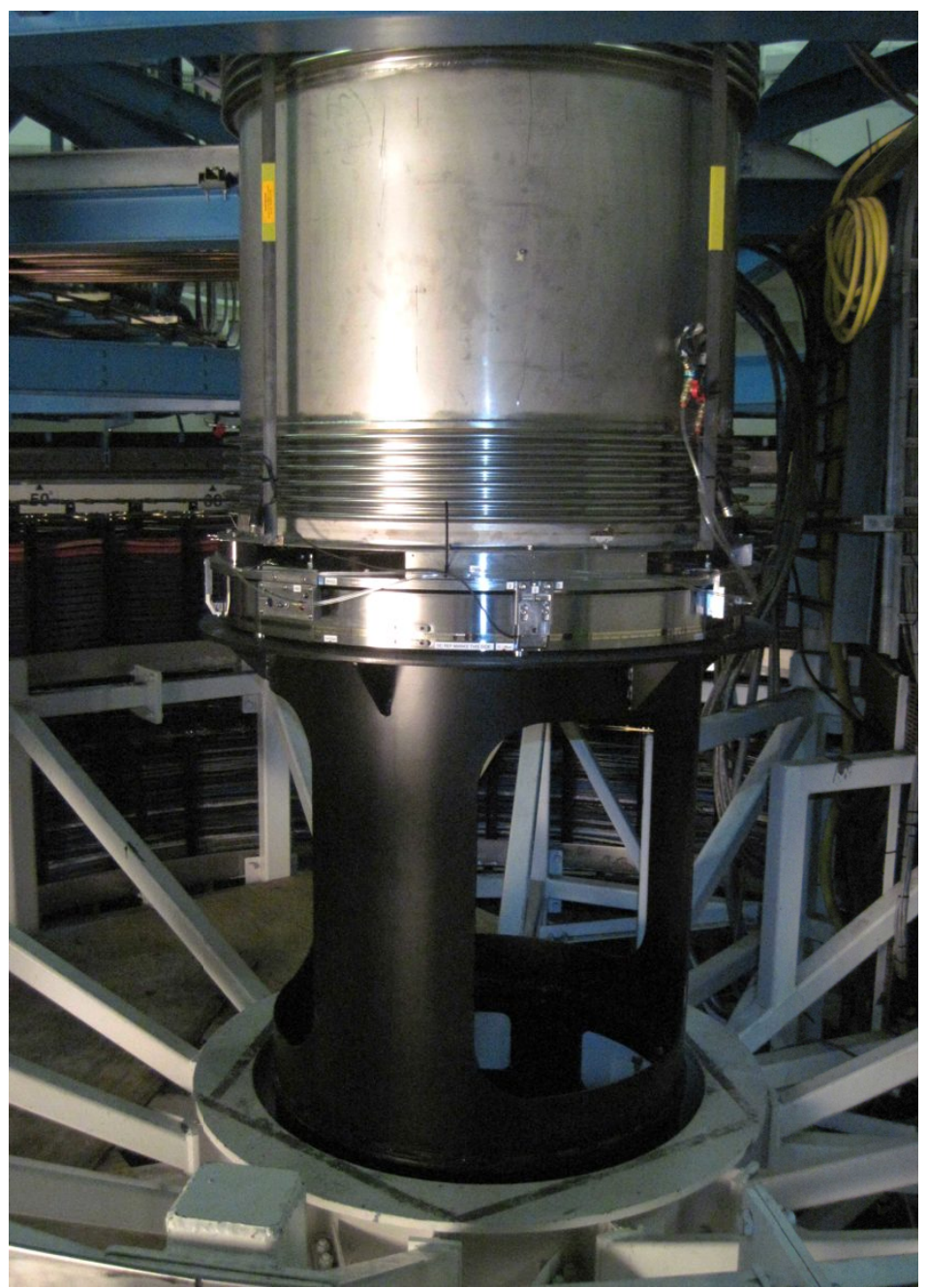

Figure 2. Azmuth bellows coupling and encoder ring installed in the Keck telescope dome at the axis of rotation.

New telescope encoders based on Heidenhain's 40 micron grading optical tape scales and tilt meters were installed to provide more accurate position sensing. The installation of these two components was a large part of the project and required a significant amount of mechanical infrastructure to house them. These two components are also the primary reason that the project achieved its tight pointing and tracking requirements.

\subsection{OH-Suppressing Infrared Imaging Spectrograph Upgrades}

Lead by PIs Dr. James Larkin and Dr. Mike Fitzgerald at the UCLA Infrared Astronomy Lab, the OH-Suppressing InfraRed Imaging Spectrograph (OSIRIS) was upgraded in two phases. OSIRIS consists of an integral field spectrograph and a separate imager, and the instrument is used in combination with the Keck I adaptive optics system to produce pointspread functions of about 50 mas. The OSIRIS spectrometer uses a lenslet array to sample a small rectangular patch of sky at spatial resolutions approaching the diffraction limit of the Keck I telescope and provides spectral resolutions of $\mathrm{R} \sim 3800$ from $\mathrm{z}$ to $\mathrm{K}$ bands. Both the spectrometer and the imager were upgraded with H2RG detectors and side car 
ASICs to improve the overall sensitivity of the instrument. Both detectors and ASICs are controlled using the Teledyne supplied software deployed on a windows operating system.

\subsubsection{OSIRIS Spectrometer}

The spectrometer upgrade ${ }^{7}$ was completed in April 2016 after four months of integration and testing during which it was unavailable to the observing community. In addition to the detector, the upgrade included new computers with Red Hat Enterprise LINUX operating systems, a focus stage, and an external detector and motor controllers. The installation was completed inside the adaptive optics clean room on the Keck I telescope.

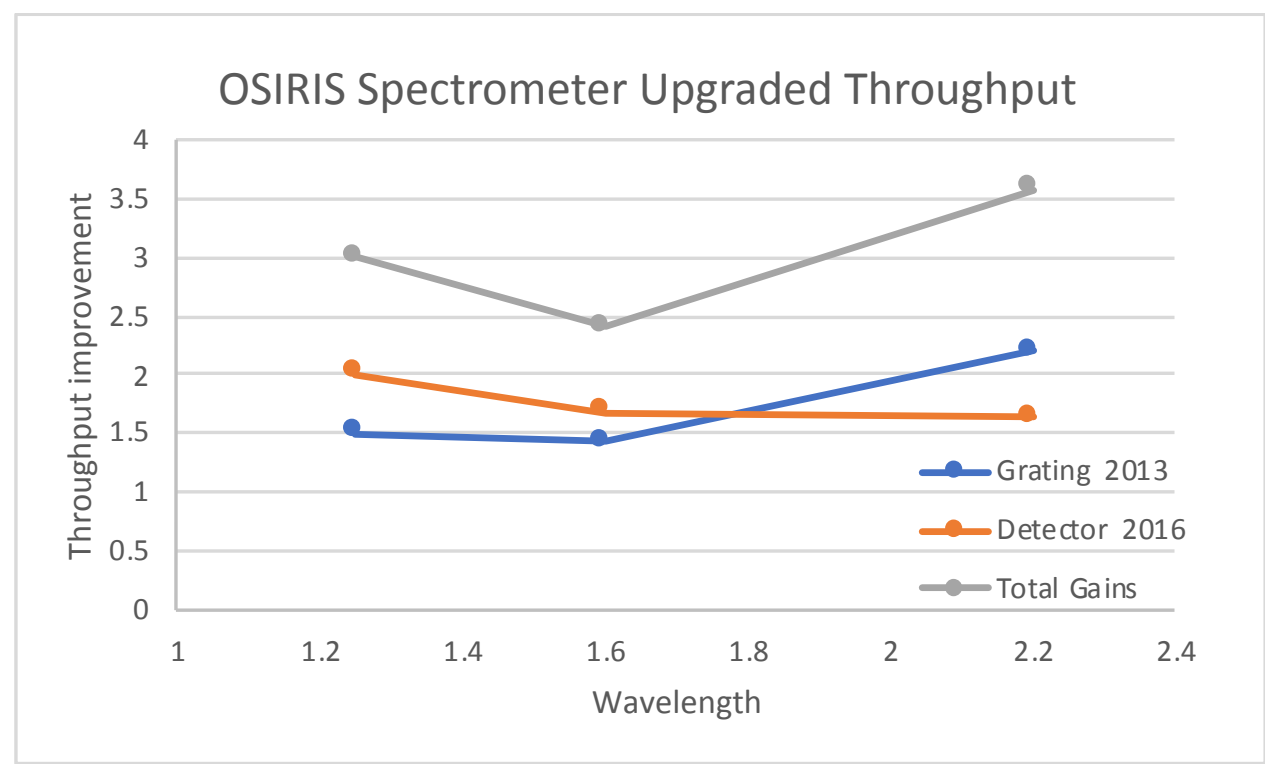

Figure 3. Upgraded throughput for the OSIRIS spectrometer. The grating upgrade completed in 2013 is multiplied by the gains in detector sensitivity to calculate the total increase in throughput.

When combined with a previous grating upgrade in 2013, the overall throughput gains are a factor of three over the original delivered instrument. The biggest sensitivity gains are in the $\mathrm{K}$ band (see figure 3 ). With the substantial increase in overall throughput, the new spectroscopic zero points were calculated to be $24.7,25.3$, and 25.1 for the $\mathrm{J}, \mathrm{H}$, and $\mathrm{K}$ band, respectively. In addition, the new H2RG has better noise characteristics and is better cosmetically with fewer pixel artifacts. The new focus stage is used to optimize the lenslet PSF shape on the detector, and the improved focus results in better spectral extraction in the post processing.

For the past two years, we focused on updating the data reduction pipeline (DRP) for use with the new detector and improving the DRP to mitigate long standing issues that were more pronounced with the new system. One of the strengths of Keck is that the observatory's community of observers are engaged in characterizing and improving the performance of the instrument suite, and the DRP improvements highlight this connection with the community. Although the upgrade required changes to accommodate the new detector, the project also provided an impetus to pursue the DRP systematics, and over the last year, Dr. Tuan Do (PI) organized a team of OSIRIS and adaptive optics users primarily at UCLA to focus on diagnostics, issues, and solutions to current problems. Three "hackathons" were held to characterize and understand the DRP issues. Two core issues were identified, and one of those issues was resolved with the upgraded detector.

The remaining issue is a flux artifact where the DRP spectral extraction routine does not properly assign flux to the correct lenslet and wavelength (see figure 4). The flux artifacts sum to zero but contributes to the noise. The leading cause is likely a mismatch between the calibration and on-sky PSF, but further investigation is needed. Through better management of bad pixels, an understanding of how to better acquire calibrations, and improvements to cosmic ray rejection routines, the changes implemented by the team improved the signal-to-noise in the spectral extractions. As part of the improvements, the team changed the distribution model for the DRP to an observatory managed github repository so that the community of OSIRIS DRP users have improved tools for testing and vetting changes before distribution. 
The DRP user group continues to be active with monthly meetings occurring at both UCLA and WMKO. For more information on the DRP see the on-line documentation ${ }^{8}$.
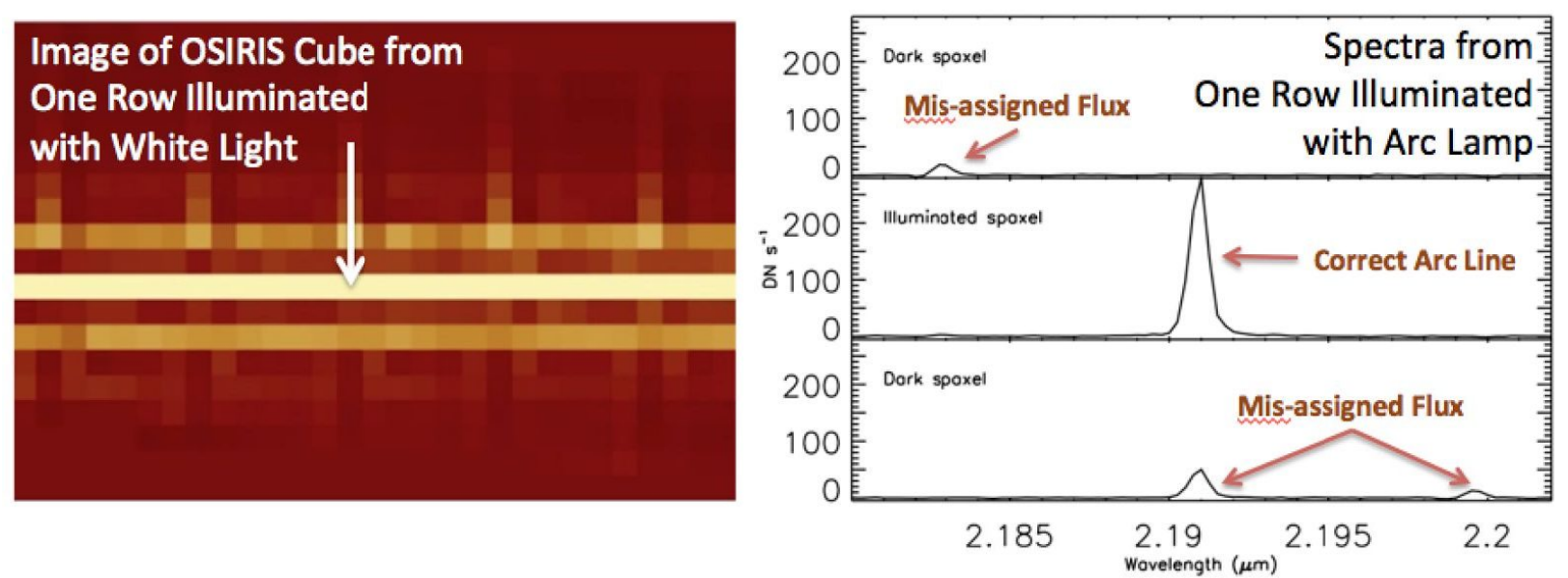

Figure 4: A single row of OSIRIS spaxels are illuminated with a source either with white light (left) or arc lamps (right); however, the extracted data cube shows evidence of flux artifacts that are present on other spaxels after DRP processing. Left: The image of the reduced data cube for a white light source illuminating a single row, showing the correct illuminated row of pixels and the flux artifacts in the adjacent rows above and below. Right: Extracted spectra from three different spaxels for an arc lamp source illuminating a single row. The illuminated spaxel shows a strong arc line. The two adjacent spaxels show flux artifacts offset in wavelength as well as spatially.

\subsubsection{OSIRIS Imager Upgrade}

In January 2018, we recommissioned the OSIRIS imager which was upgraded with a new detector, computer running RHEL, two filter wheels, a new set of optics, and associated cabling. The hardware was installed in November and adjusted in January to better align the imager with the spectrograph. To complete the upgrade, the entire optical bench was removed (see figure 5). A new optical bench was bolted into position with all the new hardware attached. A calibration source available on the AO system was used to determine the instrument focus and aligned the imager. The imager may be used on sky; however, the imager and spectrometer are currently not parfocal, and thus, may not be used simultaneously as originally designed. A service mission in early fall of 2018 will correct the parfocality. The detector performance has been verified in that lab and on sky ${ }^{9}$.
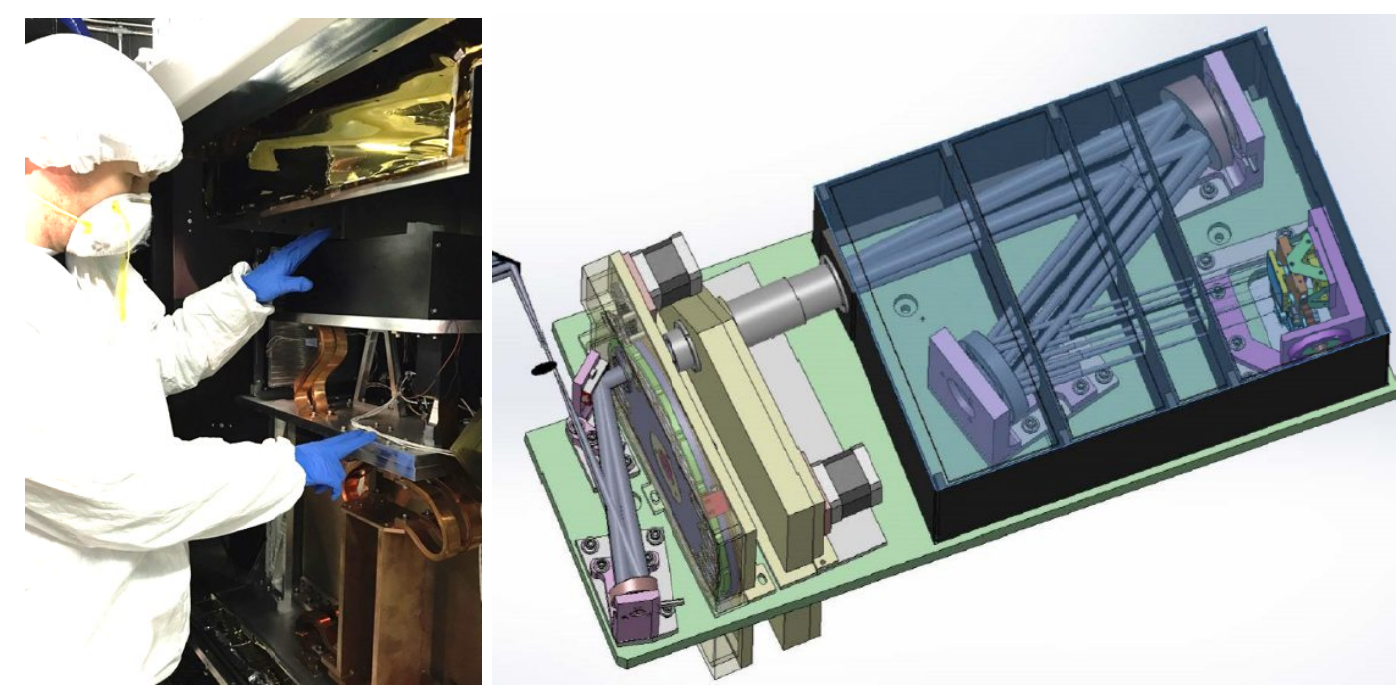

Figure 5: Dr. Jim Lyke indicating the volume of hardware replaced during the OSIRIS imager upgrade (left). The design of the optical bench is shown (right). The black enclosure on the left that has Dr. Lyke's left hand touching it is the same black enclosure in the optical design. The imager and spectrograph optical benches are side by side in OSIRIS. The first element in the optical path in the image above is the OSIRIS tent mirror which selects two fields for the two sides. 
Before the upgrade, OSIRIS was using a combination of both Solaris and Linux based computers. We took this opportunity to make all operational computers identical so that there are common accounts, communication protocols, and software deployment methods. This was not part of the upgrade but was necessary for maintaining the system into the future. These computer and software modifications are completely transparent to the user.

\subsection{Adaptive Optics system upgrades}

In the past two years, two AO project demonstrations were completed. These two modifications lead to improved Strehl ratios and a better understanding of the astrometric and photometric accuracies when using AO. These two projects are being turned into facility class additions that will benefit the broader $\mathrm{AO}$ community.

\subsubsection{Point Spread Function Reconstruction}

The Galactic Center Group (GCG) at UCLA has been testing the theory of general relativity by monitoring objects orbiting the supermassive black hole at the Galactic Center with both the Keck I and II AO systems using both OSIRIS and NIRC2. The GCG identifies variations in the PSF due to atmospheric effects and instrument aberrations as the primary source of uncertainty to both the photometry and astrometry achievable with both NIRC2 and OSIRIS. Both WMKO and the GCG have devoted effort to reconstructing and understanding the AO-corrected PSF.

Progress has been made toward implementing an on-axis PSF reconstruction (PSF-R) capability at WMKO and comparisons between the reconstructed PSFs to the measured PSF $^{10}$. Science verification results that explore the photometry and astrometry of a low-mass binary system and the morphology of QSO host galaxies indicate that the PSF$\mathrm{R}$ project has completed the demonstration phase. In this next phase, data products will be automatically provided to the AO observer and archived. The PSF-R project has been instrumental in resolving and identifying technical problems with the Keck primary mirror active control system resulting from individual segment piston errors. The PSF reconstruction project is still closely examining low order static aberrations to understand how to better correct for them.

In a parallel effort, the GCG has completed a project to predict PSF variability within Keck AO images ${ }^{11}$. They have created a new software package (AROPA) ${ }^{12}$, based on modified versions of StarFinder and Arroyo, that takes atmospheric turbulence profiles, instrumental aberration maps, and images as inputs and delivers improved photometry and astrometry.

\subsubsection{Infrared tilt compensation with adaptive optics}

The laser guide star adaptive optics (LGSAO) system on both Keck I and II has performed enormously well, but each system is limited in both performance and sky coverage because they have relied on a natural guide star (NGS) that is used for low order correction (tip-tilt and defocus modes). This limitation can be reduced by measuring image motion of the NGS in the near-infrared where it is partially corrected by the LGSAO system ${ }^{13}$. There are three potential benefits of sensing in the near-IR for LGSAO: (1) the average star is considerably brighter in the near-IR than in the visible; (2) closing the LGS loop provides sufficient correction in the near-IR to allow tip-tilt sensing on a near-diffraction limited, rather than seeing-limited, image; and (3) allowing or improving LGSAO observations of dust obscured regions. The Keck I LGSAO system now offers a choice between visible and near- infrared (IR) tip-tilt sensors: STRAP (System for Tilt Removal with Avalanche Photodiodes) and TRICK (Tilt Removal with Infrared Compensation at Keck).

TRICK uses a H2RG-based near-infrared camera with 0.05 arcsecond pixels. Low noise at high sample rates is achieved by only reading a small region of interest, from $2 \times 2$ to $16 \times 16$ pixels, centered on an NGS anywhere in 100 arcecond diameter field. The sensor operates at either Ks or H-band using light reflected by a choice of dichroic beam-splitters located in front of OSIRIS. As a demonstrator, the implementation involved modifications to the AO bench, real-time control system, and higher-level controls. Trick significantly outperforms STRAP for stars with H/Ks brighter than 14 magnitudes ( $\mathrm{R}$ brighter than $\sim 16)^{14}$.

In the past year, the TRICK control software was upgraded to make TRICK a facility class system. TRICK was more seamlessly folded into existing AO control software, and we improved automation of target acquisition, telescope offsetting, and system recovery when TRICK faults. We anticipate that TRICK will be in regular use at the observatory starting during the 2017B semester. To that end, planning tools will be provided to the observing community to select potential IR NGS stars favorable for TRICK. 


\subsection{Keck I Deployable Tertiary Mirror}

With survey telescopes such as the Zwicky Transient Factory and Pan-STARRS in operation and the Large Synoptic Survey Telescope in the pipeline, thousands of transient astronomical phenomena such as supernovae, asteroids, flaring, stellar variability, and exotic objects will be detected and will benefit from follow up observations from large groundbased observatories. To become nimbler, WMKO in partnership with UCSC developed the Keck I Deployable Tertiary Mirror (K1DM3) to prepare the observatory for more time domain astronomy observations. These types of observations included higher frequency cadence observing as well as rapid follow up of recently discovered objects. With the K1DM3 module, WMKO observers will have access to all instrument at the Nasmyth focal planes as well as a one instrument at the Cassegrain location. Newly discovered sources will require spectroscopic observations to characterize the objects or establish redshifts, and with K1DM3, both optical and infrared spectrographs will be available: OSIRIS and HIRES at Nasmyth and either MOSFIRE or LRIS at Cassegrain.

Led by Dr. J. Xavier Prochaska, K1DM3 will be able to switch between instrument focal stations in a few minutes ${ }^{15}$. The project passed a preship review in December and was delivered to WMKO in early February. From March through the summer months, both UCSC and WMKO staff have worked to integrate the module with the telescope, and this has been a challenging effort for multiple reasons. The most challenging reason is that the observatory has not shut down operations to accommodate integrating the instrument at the observatory because we strive to not impact science operations in order to maintain the observatory's scientific productivity. As a result, when the telescope is required to test functionality, complete fit checks, test communication, and resolve physical interferences, the observatory staff have to complete multiple instrument configurations and crane K1DM3 and the existing tertiary mirror to and from the floor. Sometimes this is a daily set of activities and only results in 1-3 hours of test time depending on other observatory activities required that day. Although this has lengthened the integration activities, K1DM3 will achieve first light in this summer and will be in routine operation starting in August 2018.

The current tertiary mirror was the base for the design, but K1DM3 uses a smaller mirror to make retraction from the beam practical. The retraction and deployment will be restricted to a range of telescope elevations for proper motion with favorable gravity vectors. While the Keck telescopes support a 20' of view (FOV), the K1DM3 supports an unvignetted FOV of $4.7^{\prime}$ which will work well for the smaller fields needed for both HIRES and OSIRIS at the two Nasmyth focal stations. The focal plane will remain unvignetted at the Cassegrain focal station. Once commissioned, K1DM3 module will remain in the telescope at all times.

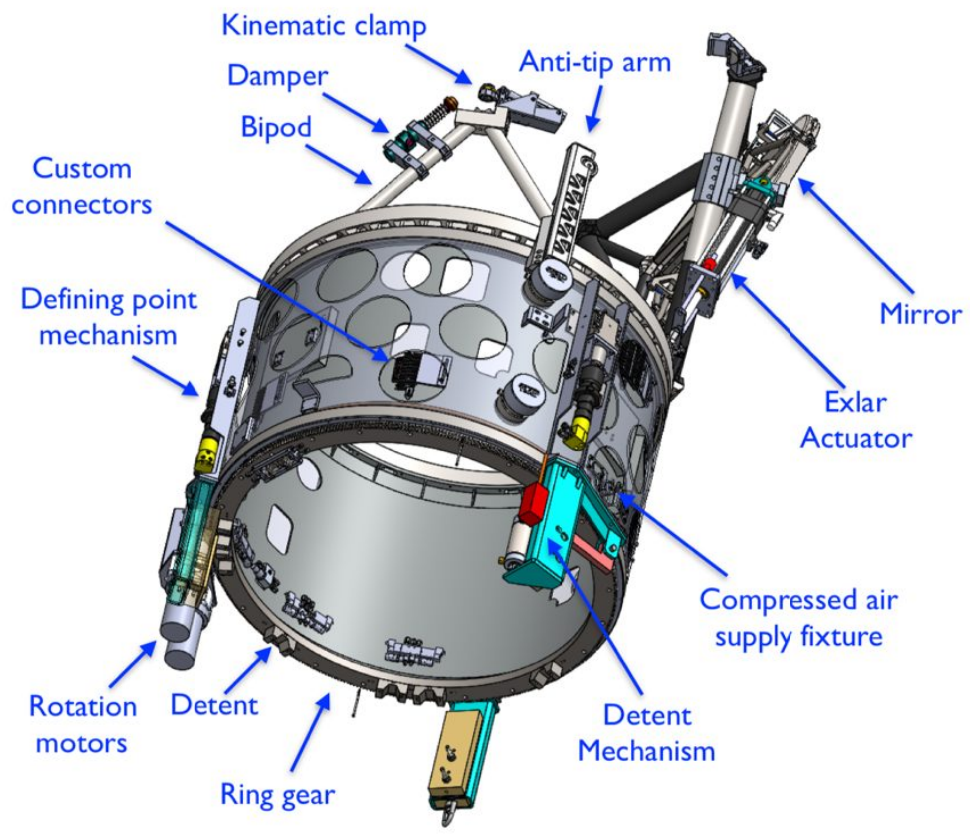

Figure 6: K1DM3 viewed from the back with various components labeled. 


\section{UPGRADES AND NEW INSTRUMENTATION IN PROGRESS}

\subsection{Near IR echelle spectrograph upgrade}

The near-IR echelle spectrograph, NIRSPEC ${ }^{16}$, is a workhorse facility instrument for the Keck II telescope that is optimized for high-resolution R 25,000 spectroscopy at wavelengths of 0.95-5.5 microns. An upgrade currently in progress will provide enhanced and new capabilities, and increased sensitivity for a productive and popular instrument which will extend its lifetime significantly ${ }^{17}$. Led by Dr. Mike Fitzgerald at UCLA, the upgrade includes two H2RG detectors one for the spectrometer and one for the slit-guiding camera (SCAM), new optics for SCAM, a new filter wheel for SCAM, new computers, and new motor and detector controllers. The upgraded detector on the spectrometer will have a reduction in readnoise that is a five times improvement over the existing detector and will exhibit less dark current by a factor of 70. In addition, the smaller pixels will increase spectral resolution from 25,000 (0.43" slit, $>$-pixel sampling to 37,500 (0.29" slit, 4-pixel sampling).

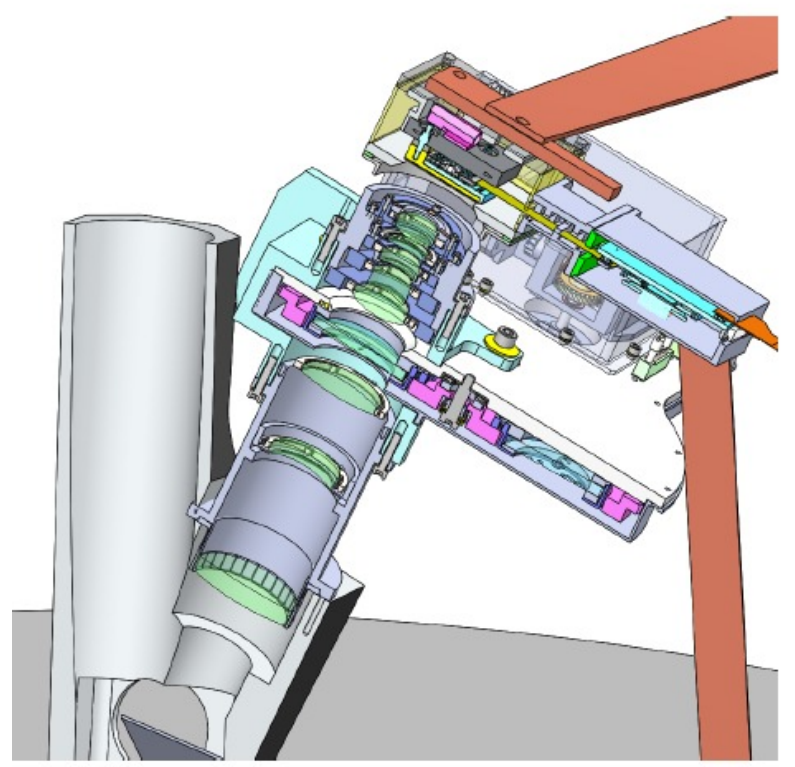

Figure 7: Image of the new SCAM hardware including the optics, filter wheel, and detector installed in all new mechanical assembly.

The upgrade will not only make NIRSPEC more sensitive with advanced detector technology, but it also provides new capabilities and retires obsolescence issues. The SCAM optics upgrade is a key component to this as it will expand guiding into the thermal infrared to match the extent of the science wavelength coverage. The current guider may only be used for on-slit guiding at wavelengths shorter than $\mathrm{K}$ band. The new optics and detector extend the wavelength range and provide the necessary filters for the longer wavelengths. We are also replacing obsolete transputers used for motor and detector controllers with modern detector systems identical to those now in use with OSIRIS and Galil motor controls that are in use many observatory instruments. To retire risk and reduce down time, the electronics were fasttracked and successfully tested with NIRSPEC in early April.

The project passed a preliminary design review in mid-November 2017 and is currently in a full-scale development with delivery to WMKO scheduled for early August. An all new mechanical assembly for the lenses and filter wheel is under fabrication and is scheduled to be completed in time for the optics delivery in mid-June 2018. Lab testing will be completed in July before shipping in early Aug 2018. The instrument will be unavailable for use from 1 August to 9 December 2018 when the new components will be integrated and tested in the instrument. NIRSPEC will be returned to operations in time for the NASA community to observe comet Wirtanen that will pass within 30 lunar distances of Earth and reach an estimated brightness of 3-7.5 magnitudes. This close flyby gives comet observers an opportunity to study and map different compounds in the comet that they might not have detected before and NIRSPEC is an optimal instrument for completing these studies. 


\subsection{Keck Cosmic Reionization Mattper}

Following delivery of KCWI and its transition to routine observing ${ }^{2,3}$, the development team turned its attention to the Keck Cosmic Reionization Mapper (KCRM) that is the red half of KCWI. Led by Dr. Christopher Martin, KCRM is a collaboration between Caltech, UCSC, and WMKO and is scheduled for delivery to the observatory in December 2020 with first light in summer of 2021.

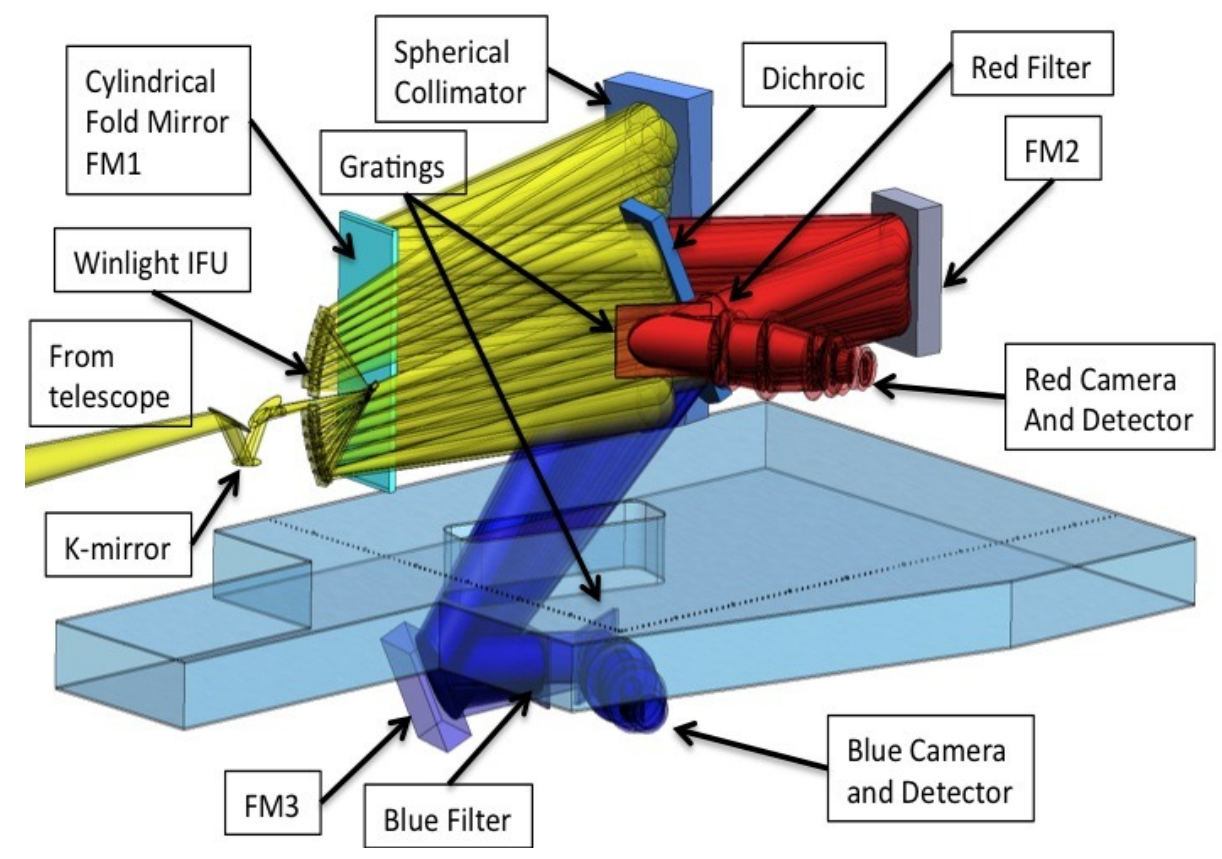

Figure 8: The optical layout of KCWI with the red arm, KCRM, highlighted in red. The light blue represents the optical bench and while KCRM is bolted to the top of the bench, the blue side hangs from below. The dichroic in the diagram is currently a fold mirror directing all light to the blue side. The guider is not shown.

KCRM will employ a high efficiency, red optimized CCD detector, configurable high spectral resolution, and superb sky-subtraction to create a unique and powerful capability ideally suited to studying the reionization of the Universe. The red detector will be a Lawrence Berkeley National Laboratory (LBNL) 4k x 4k, 500 micron Deep Depletion CCD (DDCCD) with 15 micron square pixels that will have high quantum efficiency and extend the wavelength coverage from 0.560 to 1.05 microns. The detector will be implemented by UCSC who were responsible for the 2010 upgrade to the red channel of WMKO's LRIS instrument using DDCCDs developed by LBNL. Several different gratings will be available for use during the night at both low and high resolutions (R 1,000-20,000), and both the blue and red side will be able to operate simultaneously to obtain data on the same source.

The current guider in KCWI as well as an existing fold mirror require modifications in order to accommodate KCRM. The current guider picks off light that would nominally go to KCRM's science detector while a mirror directs all light to the blue sensitive detector. With KCRM, we will replace the guider with a system that will pick off an annular field in front of the K-mirror, and the current fold mirror will be replaced with large $250 \times 700 \mathrm{~mm}$ dichroic made of fused silica (see figure 8). The blue light path is otherwise unmodified, and the new dichroic improves red rejection on the blue side.

Cost reviews were held in June 2017 and January 2018 with lessons learned and actual costs from the blue side applied to the budget and schedule for KCRM. The preliminary design phase was started in February and a preliminary design review is scheduled for October 2018. Some of the KCRM components are copies of the blue side (e.g. articulation stage), and thus the costs for some sub components are very well defined.

\subsection{Keck Planet Finder}

The Keck Planet Finder (KPF) ${ }^{18}$ is a fiber-fed, high-resolution, high-stability spectrometer in development for the Keck I telescope. The instrument is a collaboration between the SSL, Caltech, WMKO, UCSC, and Macquarie University being led by Prof. Andrew Howard at Caltech. The instrument is designed to characterize exoplanets via Doppler 
spectroscopy with a single measurement precision of $0.5 \mathrm{~m} / \mathrm{s}$ or better. Its resolution and stability will enable a wide variety of astrophysical pursuits. KPF will have a $200 \mathrm{~mm}$ collimated beam diameter and a resolving power of $>80,000$. The design includes a green channel $(445 \mathrm{~nm}$ to $600 \mathrm{~nm})$ and red channel $(600 \mathrm{~nm}$ to $870 \mathrm{~nm})$, each optimized for their respective bandpasses. This next generation precision radial velocity instrument will be significant step forward in spectral resolution, sensitivity, speed, and precision and will help maintain WMKO's lead role in exoplanet discovery, confirmation, and characterization.

At a basic level, KPF is an optical echelle spectrometer, but achieving the highest spectral sensitivity requires KPF to incorporate many state-of-the art technologies. A novel design aspect of KPF is the use of a Zerodur optical bench and Zerodur optics with integral mounts to provide stability against thermal expansion and contraction effects. In addition to Zerodur's stability, the KPF team will take advantage of design concepts and sub-systems that have proven to stabilize instruments for exoplanet work, and a recent preliminary design review panel commended the team for using many recent advancements in the field.

The design of the instrument includes:

- A mechanical agitator that physically moves the fibers to suppress quasi-static speckle patterns (modal noise) exhibited by stationary fibers.

- An optical fiber double scrambler that homogenizes the beam by exchanging the near and far-fields between two octagonal fibers.

- A large echelle mosaic grating that disperses the light into a spectrum.

- A VPH gratings for tailored cross-dispersion to fit the spectra on a pair of 4k x 4k CCDs.

- A custom mirror assembly called the "reformatter" that maps the light from an optical fiber, slices the light, and stacks it in a pseudo-slit to reach the desired spectral resolution.

- Simultaneous calibration fiber and a suite of calibration sources including an $\mathrm{Rb}$-stabilized etalon for precise wavelength calibration that will accurately track instrumental drift.

- A supplementary UV spectrometer (385-405 nm) that will capture the Ca II H \& K lines at low resolution to monitor stellar magnetic activity.

KPF is currently in the detailed design phase having successfully passed its preliminary design review in November 2017. Two major changes to the plan from the observatory's perspective are that the instrument's fiber injection unit (FIU) is now planned for installation in the Keck I adaptive optics enclosure and the spectrometer will be placed at the end of the long delay line hallway in the basement. This provides increased stability for the FIU, and significantly decreases the fiber length, improving the spectrometer's throughput at the bluest wavelengths.

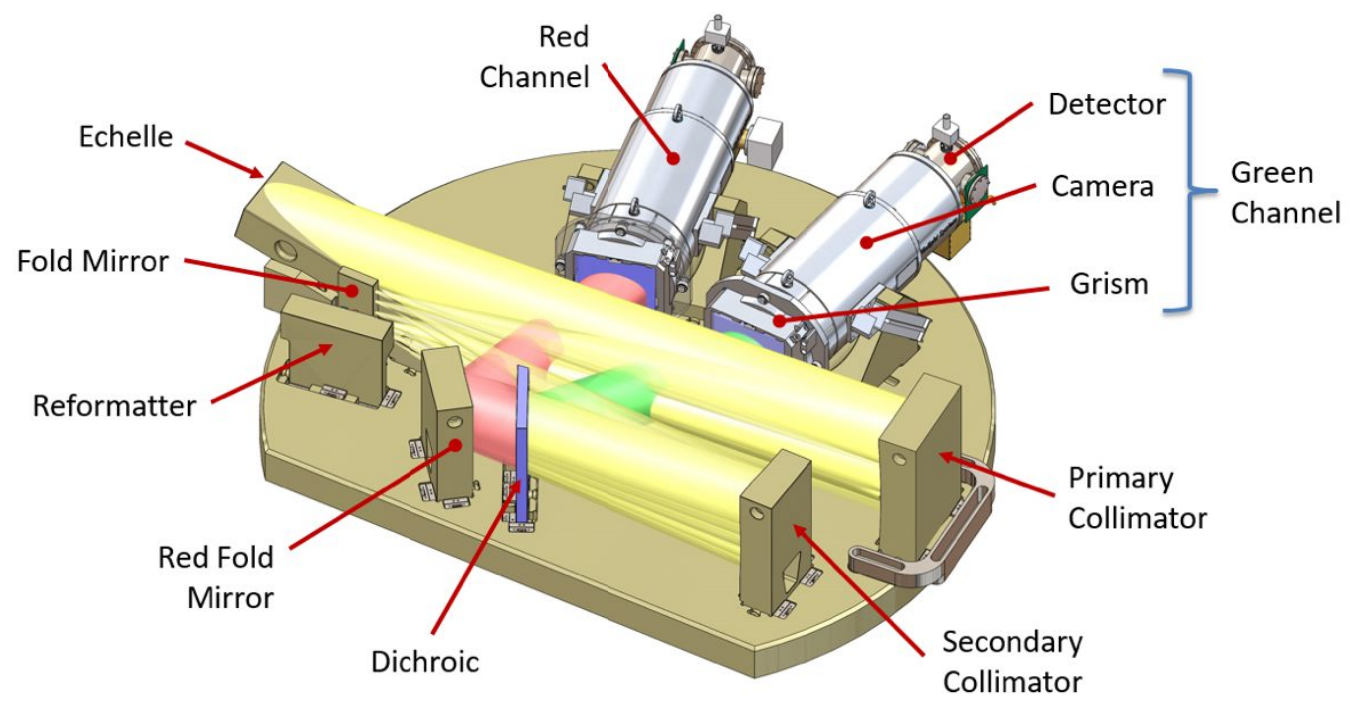

Figure 9: The conceptual opto-mechanical design for KPF. 


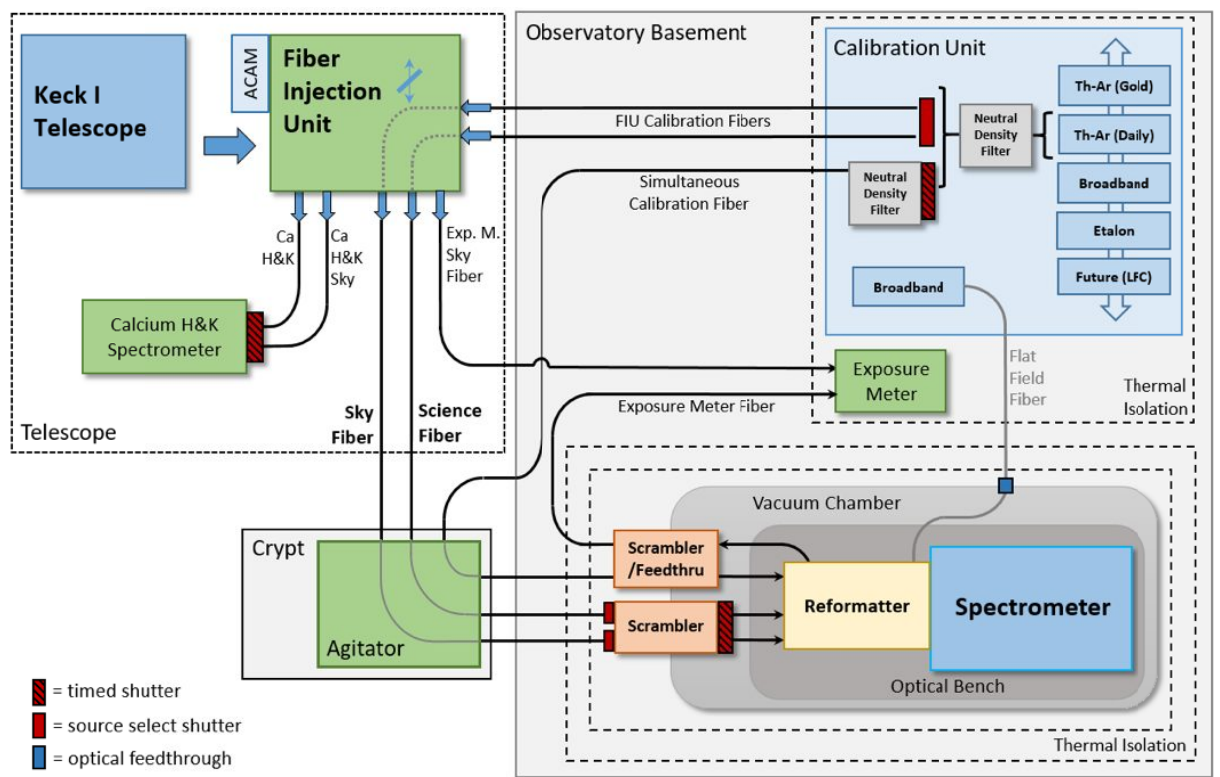

Figure 10. The KPF system overview with observatory locations labeled. Telescope refers to the Keck I AO enclosure, crypt represents the room in the basement directly below the Keck I telescope, and the basement is the end of the long hallway close to Keck I.

\subsection{Adaptive Optics projects}

Below we briefly describe the current AO development projects that are in progress. These projects include advances to the existing AO systems as well as demonstrator hardware and software that we plan to turn into facility class systems in the future. These three separate AO technology demonstrators when combined can achieve new science at the observatory.

\subsubsection{Real time wave front controller}

In partnership with Microgate in association with Swinburne, ANU and Observatoire de Paris, we are upgrading the existing wave front camera and controller ${ }^{19}$ on the Keck II AO system. The upgrade builds on recent success in implementing of a sodium-wavelength fiber laser and the launch of this laser from behind the Keck II telescope secondary mirror, replacing the first-generation side launched laser.

The new real-time control system will help support the new near-infrared pyramid wavefront sensor (see section 3.4.2). Because, the new real-time computer has to interface with an existing and very productive adaptive optics system, we have elected to implement the new hardware and control system without impacting our science operations during the development or deployment. Our system is based on the independent Subaru Coronagraphic Extreme Adaptive Optics pyramid wavefront sensor real-time processor solution that will use the hardware interfaces provided by the existing Keck II real-time controller. We anticipate that the new hardware will provide a one magnitude sensitivity gain and allow the telescope to perform near its diffraction limit.

\subsubsection{Pyramid Wavefront Sensor}

This fall, we are implementing a near-infrared, high order pyramid wavefront sensor on the Keck II telescope ${ }^{20,21}$, with the aim of providing high resolution adaptive optics correction for the study of exoplanets around M-type stars and planet formation in obscured star forming regions. The pyramid wavefront sensor is designed to support adaptive optics correction of the light to an imaging vortex coronagraph installed in the second generation Near Infrared Camera (NIRC2) and to a fiber injection unit that will feed the near infrared, high-resolution spectrograph NIRSPEC undergoing upgrades (see section 3.1). The hardware is installed on the AO bench near the entrance of the NIRC2 instrument. We completed the opto-mechanical design of the near-infrared pyramid wavefront sensor, characterized the optical performance, and developed an alignment strategy which is complicated by the fact that the Keck II AO bench has limited space available ${ }^{20}$. In the 2019 observing semesters, we will be completing science verification of this new capability by observing many exoplanet systems. 


\subsubsection{Fiber Injection Unit with AO}

In collaboration with Caltech, JPL, UCLA, WMKO is installing a demonstrator Fiber Injection Unit (FIU) on the Keck II AO bench that will fiber feed NIRSPEC to develop and test an IR precision radial velocity ${ }^{22}$ capability. The NIRSPEC upgrade will increase the spectral resolution, and the addition of the FIU will stabilize the PSF on the NIRSPEC slit. In combination with the pyramid wavefront sensor, we hope to demonstrate that WMKO can achieve high resolution adaptive optics correction for the study of exoplanets around M-type stars and planet formation in obscured star forming regions. Thermal emission from young giant planets will be injected into a single mode fiber linked to NIRSPEC thereby allowing spectral characterization of exoplanet atmospheres.

Caltech engineers completed an opto-mechanical design of the FIU and supporting hardware on NIRSPEC, passed a detailed design review, and will install the hardware for initial testing late this summer and early fall. The FIU was integrated with the Pyramid WFS at the Institute for Astronomy in Hilo in May 2018, and the team is completing system level testing. There is limited space available on both the AO bench and on board NIRSPEC that the design team needed to accommodate. Science schedule pressures and higher profile projects have delayed installation. Due to the NIRSPEC upgrade discussed in section 3.1, on sky testing will start following the return of NIRSPEC to routine operations in midDecember.

\section{FUTURE INSTRUMENTATION PROJECTS}

To remain competitive in an ever-evolving landscape of scientific interests and technology advances, WMKO in partnership with its SSC annually solicit instrumentation concepts from the community. Through a white paper call sent in the spring, the SSC advertises needs defined in the strategic plan, and the community proposes new facility class instrumentation, upgrades to existing instruments, or enhancements for aging instruments and observatory systems. For all projects, the community must seek approval from the SSC before moving forward with designs and construction.

This year, WMKO with approval and input from the SSC accepted two types of proposals, conceptual designs as well as Phase A system design proposals. Conceptual designs seek to grow interest in the proposed instrument, develop the initial science cases, and identify technical advances the instrument will have. Phase A system designs are funded at a higher level and are to develop more detailed instrument designs, grow the science cases, and develop a robust schedule and budget for the delivery of the instrument. At the end of a year, the development teams report back to the SSC for approval to move to the next phase or to seek funding.

Top priorities identified in the strategic plan include a multi-object spectrograph capable of sampling most of the 20 arcmin Keck telescope focal plane and a ground layer adaptive optics (GLAO) system. These two community driven desires are currently in conceptual design phase. In addition to these two major facility class initiatives, there are a number of other proposed projects large and small that are in conceptual and system design phases.

\subsection{Ground Layer Adaptive Optics}

The atmospheric profile above Maunakea is well suited to providing seeing improvements with GLAO which is identified by the community as a priority for enhancing WMKO's competitiveness. GLAO has the potential of providing partial correction of atmospheric blurring over a significantly larger field of view and over a broader wavelength range than Keck's existing AO systems. With an expected 2x better spatial resolution in the near-infrared and red-optical, GLAO should benefit all seeing limited instruments, but in particular, the community see significant improvements for large field multi-object spectrographs, DEIMOS and LRIS at optical wavelengths and MOSFIRE in the infrared. Science applications enabled by GLAO are thus complementary to classical AO and include extra-galactic spectroscopic surveys over a broad range of redshifts using MOSFIRE, LRIS, and DEIMOS, intergalactic and circumgalactic medium studies with integral field and slit spectrographs like KCWI and LRIS, and stellar population studies in the Milky Way and nearby galaxies where crowding limits current sensitivities.

A GLAO conceptual design study is being led by Dr. Jessica Lu at UC Berkeley and Dr. Mark Chun at the University of Hawaii. The study is now starting year three as they continue to determine feasibility for the WMKO's instrumentation suite and telescope. This team is applying what they have learned from their pilot program called 'imaka that is a GLAO demonstrator with an $18 \times 24$ field of view on the University of Hawaii $2.2 \mathrm{~m}$ telescope ${ }^{23,24}$. Results from 'imaka indicate that the PSF FWHM is improved by a factor of $\geq 1.4$ and the noise equivalent area is improved by better than a factor of two. The team believes that the improved performance is both uniform and stable and will be beneficial for multi-object spectrographs at WMKO. 
The team has been examining the intrinsic image quality of the telescope and the instruments to combine these factors with a calibrated atmospheric model. Multiple atmospheric models are being tested to confirm that the results are consistent. The team is demonstrating that most of the existing WMKO instruments can benefit from GLAO improvements.

\subsection{Fiber-Optic Broadband Optical Spectrograph}

Currently in a conceptual design phase developing science and technical cases, the Fiber-Optic Broadband Optical Spectrograph (FOBOS) represents a leap for WMKO into fiber fed multi-object spectroscopy. Primarily led by staff at UCO and Berkeley, the development team held workshops at LBL on 18-19 January 2018 and at UCLA on 4 May 2018 to garner interest and understand the science community needs. The instrument is identified in the 2016 WMKO Strategic Plan by specifically calling out highly multiplexed, highly sensitive spectroscopy by doubling the field of one of WMKO's current spectrographs DEIMOS, with a goal of supporting synergistic activities with WFIRST, Euclid, and TMT to push the limits of faint multi-object spectroscopy.
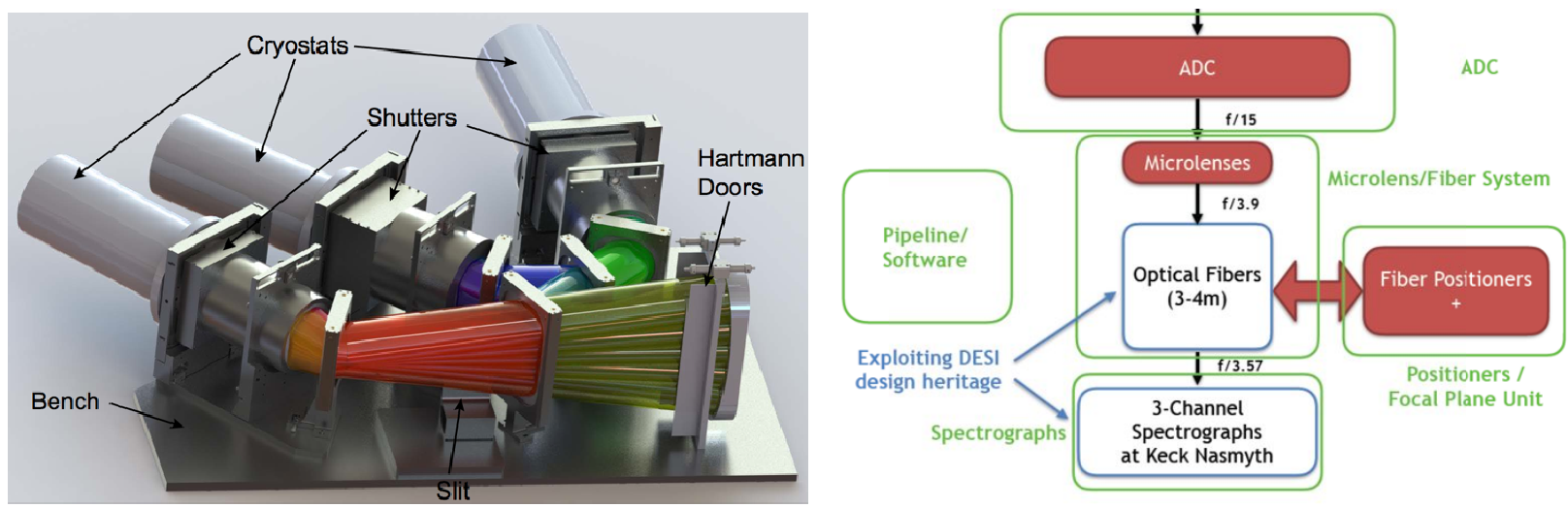

Figure 11: The conceptual design for the three-channel spectrograph based on the DESI modular spectrographs (left). The current conceptual design considers four of these in use with FOBOS. A system flow chart is presented (right). Light from the telescope enters the spectrograph from the top wheren it first passes through an ADC. The focal plane is reformated using robotic fiber positioners, and a short fiber run leads to the multiple three channel spectrographs. In this image, the instrument is located on the Keck Nasmyth location similar to the DEIMOS multi-object spectrograph.

Having a heritage from the designs of $\mathrm{WFOS}^{25,26}$ and $\mathrm{DESI}^{27}$, FOBOS is proposed as a 400 -object fiber fed high throughput spectrometer sampling the full 20 arcmin WMKO focal plane, providing medium resolution ( $\mathrm{r} \sim 2500-5000)$ spectroscopy spanning wavelengths from $350-980 \mathrm{~nm}$. The spectrograph is designed to be modular with the fibers feeding several small spectrograph units that are designed for high throughput (average $70 \%$ after fiber loses) over the three possible spectral channels 350-560, 560-750, and 750-980 nm. It will have a FOV coverage of $0.08 \mathrm{deg}^{2}$, with a target density of 2.4/arcmin ${ }^{2}$, nearly a factor of two improvement over both MOS optical spectrographs at WMKO.

FOBOS leverages technical developments in current fiber-based spectrograph and has synergies with ongoing instrumentation development at both UCO and LBL. The fiber technology is currently based on the DESI spectrograph design. The DESI design used 107 micron fibers injected at $\mathrm{f} / 3.9$ which corresponds to a 0.57 arcsec aperture when matched to the Keck f/15 focal planes with micro lenses. Because this a small aperture for individual fibers, the design team is considering mini-IFUs, fiber bundles comprised of 7x0.46 arcsec hexagonal micro lens arrays for each target, but larger IFU configurations are also under consideration.

The FOBOS team is interested in technologies that improve the blue throughput, throughput stability, and uniformity of the near/far-field illumination from fibers. The team is also exploring detector technology that could extend the sensitivity of the spectrograph beyond one micron. 


\subsection{NIRC2 upgrades}

The ability to detect self-luminous exoplanets depends strongly on the temperature of the exoplanet. In the near infrared (JHK bands), the sky background is low, but the planet-to-star brightness heavily favors the star. Although the sky brightness is higher in the thermal infrared (3-5 microns), the planet is also brighter relative to the star, and thus, there is a significant advantage in taking a direct image of exoplanets at longer wavelengths. In recent studies, planets may be directly imaged in the thermal infrared but may remain undetected at shorter wavelengths (1-3 microns).

Led by Dr. Dimitri Mawet at Caltech, we intend to leverage the capacity of the workhorse instrument NIRC2 to detect thermal emission by upgrading NIRC2 with an M-band vector vortex coronagraph. The new vector vortex technology enables high contrast imaging of faint targets by suppressing the light from a central star to directly image exoplanet. To further improve our detection limits, we will fabricate and add a new Lyot stop to mitigate systematic noise from light diffracted by the telescope and $\mathrm{AO}$ system, and fabricate and install new mechanical mounts for proper positioning of the vector vortex coronagraph. We anticipate that these modest hardware and software upgrades will be completed during the next year and are designed to take advantage of the advances made through the addition of the Pyramid Wavefront sensor that will deliver an infrared corrected beam to NIRC2.

In subsequent years, we hope to upgrade NIRC2 with a new set of control electronics along with upgrades to the host computers. The current control electronics by INMOS Transputers are reliable and have served the instrument well, but they are obsolete. They have long been out of production and have no real programming base. Due to overheads in readout, the efficiencies are low resulting in difficulty in observing in the thermal infrared where short exposures are desirable. In the M band, the system efficiency is only about $30 \%(50 \%$ in $\mathrm{L})$. We anticipate modernizing the detector controller at a modest cost to improve the observing efficiency numbers which could lead to doubling the productivity.

\subsection{Keck Radiometer Array using KID ENergy Sensors}

Microwave Kinetic Inductance Detectors (MKIDS) are superconducting detector arrays that can measure the energy and arrival time of individual optical and near-infrared photons without false counts. The development team at UC Santa Barbara led by Dr. Ben Mazin is seeking to take the MKID technology that they have developed for the Palomar, Lick, and Subaru Telescopes and provide a facility class instrument for the Keck I Telescope. This new instrument, the Keck Radiometer Array using KID ENergy Sensors ${ }^{28}$ (KRAKENS), will be a 30 kpix integral field spectrograph (IFS) with a 42.5 " $\mathrm{x} 45$ " field of view, wide wavelength coverage from $380-1350 \mathrm{~nm}$, and a spectral resolution $\mathrm{R}=\lambda / \Delta \lambda>20$ at 400 $\mathrm{nm}$.

A significant part of the science impact will come from the inherent time domain capabilities of MKIDs. MKIDs have an effective readout rate (sub-ms) at least 104 times faster than any other science camera at Keck, allowing them to capture time domain information on rapidly changing sources like electromagnetic counterparts to LIGO detections, optical pulsars, and exoplanet transits. In combination with the new NSF funded Keck I deployable tertiary mirror (see section 2.6), KRAKENS will open up fast time domain and cadence observations at Keck just as large synoptic surveys like ZTF and later when LSST come online.

It is anticipated that KRAKENS will also be the most sensitive instrument on Keck for measuring the SEDs of faint low surface brightness objects. For these objects, such as $\mathrm{z}>8$ galaxy candidates, the low spectral resolution is not a disadvantage since there are not enough photons for higher resolution spectroscopy. The high throughput of KRAKENS, combined with the lack of a slit, and the ability in software to dynamically vary an aperture to correct for tip/tilt should make KRAKENS at least a magnitude more sensitive than LRIS.

\subsection{Second generation integral field spectrograph for adaptive optics}

By heavily leveraging their experience building OSIRIS for Keck and developing designs for the TMT/IRIS instrument $^{29}$, Dr. James Larkin and his team at UCLA hope to build a second-generation integral field spectrograph (IFS) that would take advantage of the availability of large format Hawaii-4RG detectors and improved Keck AO performance. This instrument is currently called LIGER. The IRIS team has completed optical and mechanical designs for cryogenic collimator and camera optics that are needed for both slicer and lenslet IFS backed by larger Hawaii-4RG detectors (see figure 12). These designs have the ability to be used without significant modification for any diffractionlimited IFS with sampling scales matched to a particular telescope diameter.

A component of the new IFS would reuse the newly upgraded diffraction-limited OSIRIS imager developed at UCLA and commissioned at WMKO (see section 2.4.2). The OSIRIS imager has an ideal sampling of 10 mas per pixel and a 20 
arcsecond FOV already intended to work with increased AO performance. Sitting on a separate optical bench, it will be straightforward to move the new imager inside the new IFS dewar for a powerful parallel imaging mode.

This year the team has focused on design choices and determining scientific merits that realize the goal of a wide-field (up to 5 arcsec) IFS that is called out in the Keck AO Strategic Plan. As part of the conceptual study, the team is balancing the overall instrument cost vs. capabilities. For instance, the number of sampling scales, number of spectral modes (including range of resolutions) must be balanced with the desire to have large areal coverage ( $\sim 4$ times the areal coverage of OSIRIS). We will also explore extending the sensitivity of this IFS to optical wavelengths at least as short as 0.84 microns since the IRIS designs are already optimized for this and AO is making steady performance improvements at progressively shorter wavelengths. We will study a range of costed capabilities with their respective scientific impacts with expected gains in sensitivity from improved AO performance and the IRIS-like use of multiple diffraction gratings, each used exclusively in the first order (as distinct from the single fixed grating used in multiple orders in OSIRIS).

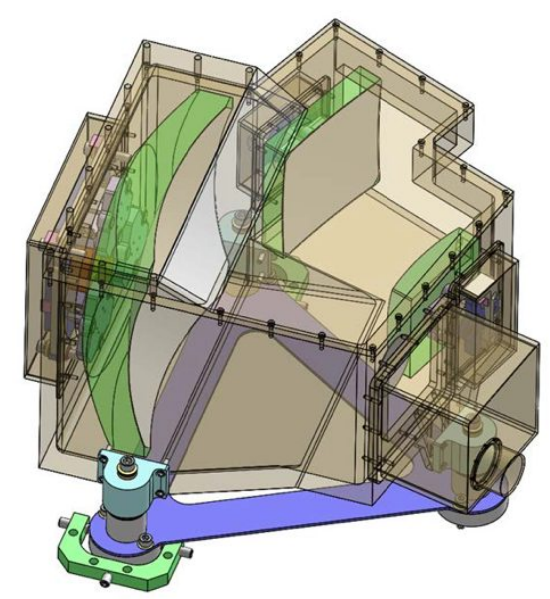

Figure 12: The opto-mechanical assembly of the camera three mirror anastigmat for the IRIS spectrograph. The large format of the detector combined with large spectral bandpass and desire for high throughput made these all reflective optical systems the design of choice. With high order aspheric surfaces, their design (by Renate Kupke at UCSC) and mechanical mounting structure (primarily Evan Kress at UCLA) are the result of a large investment by the TMT project. In a Keck instrument, they can be used basically as designed. The camera TMA shown is approximately $600 \mathrm{~mm}$ on a side and $400 \mathrm{~mm}$ tall.

\subsection{Adaptive Optics Array of Lenslets Integral Field Spectrograph}

The Santa Cruz Array of Lenslets for Exoplanet Spectroscopy (SCALES) is a proposed 2-5 micron adaptive optics integral field spectrograph for Keck. Its primary goal is to extend the wavelength range with which we can characterize directly imaged planets into the thermal infrared (L- and M-band), where self-luminous gas-giants peak in brightness. However, it will benefit all scientific areas that can make use of high angular resolution thermal infrared imaging and spectroscopy (e.g. Solar System atmospheres, Io volcanism, organics in comets, ice lines in circumstellar disks, dust streamers in interacting binaries, spatially resolved spectra of the centers of dusty galaxies).

SCALES will initially feature a $1.8 \times 1.8$ " field-of-view lenslet-based integral field spectrograph, with spectral resolutions of R 100-200 in selectable bandpasses. There will be an R 5000 mode for a smaller $0.22 \times 0.22$ " field-of-view. SCALES is designed to accept several pre-planned upgrades, including an imaging channel, a 70\% larger field-of-view for integral field spectroscopy, a speckle-nulling focal plane wavefront sensor, and a single mode fiber pickoff for highresolution spectroscopy after SCALES's cryogenic coronagraph. SCALES will be a predecessor to the TMT's Planetary System's Imager Red channel ${ }^{30}$ (PSI-red) and will play a key role in developing PSI-Red's science and user community. One of the challenges for the instrument will identifying cryogenic single-mode fiber and anticipate requiring fluoride fibers that can survive and function cryogenically.

\subsection{Keck All sky Precision Adaptive optics}

The Keck Observatory strategic plan recognizes WMKO as a leader in observations made with the adaptive optics (AO), and have identified that the science will benefit from expanded AO corrected FOVs that are larger than currently supported. WMKO is thus working towards developing the Keck All sky Precision Adaptive optics (KAPA) system to 
carry out four high impact science projects: constraining dark matter, the Hubble constant and dark energy via strong gravitational lensing; testing General Relativity and understanding supermassive black hole interactions at the Galactic Center; characterizing galaxy kinematics and metallicity in rare highly magnified galaxies; and characterizing gas-giant protoplanets around the youngest low mass stars via direct imaging and spectroscopy. The proposed science projects require $\mathrm{AO}$, an essential technology that delivers on the full potential of large ground-based telescopes and build on scientific successes using the W. M. Keck Observatory (WMKO) AO systems.

WMKO has led the field by pioneering the first natural guide star AO and the first laser guide star (LGS) AO on 8 to 10 $\mathrm{m}$ telescopes. However, current LGS AO systems achieve their best performance over only a modest fraction of the sky and with image quality considerably poorer than the fundamental limit. WMKO is seeking to add a laser capable of being split into three beacons for atmospheric tomography, enhanced near-infrared tip-tilt sensing, and point spread function reconstruction (PSF-R) to the Keck I LGS AO facility. The resulting KAPA system will have dramatically higher sky coverage with high Strehl, diffraction-limited science than any of today's AO systems. Data collected with KAPA are expected to provide a strong base for additional advances by the broader community and to prepare the community for science with LGSAO on the coming generation of extremely large telescopes.

\section{MOSFIRE RECOVERY MISSION}

MOSFIRE is the multi-object spectrograph and wide-field infrared camera at the W. M. Keck Observatory. This large vacuum-cryogenic instrument is located in a rotating module at the Keck I Cassegrain focus. MOSFIRE uses a cryogenic Configurable Slit Unit (CSU) developed in collaboration with the Swiss Center for Electronics and Microtechnology $(\mathrm{CSEM})^{1}$. The CSU allows observers to create slit masks over the $6.1^{\prime}$ x $6.1^{\prime}$ field of view (FOV) using 46 pairs of bars that move in from each side and typically form a $0.7^{\prime \prime}$ ( $\sim 3$ pixels) slit on the sky resulting in an R 3500 for spectroscopy in the Y, J, H or K bands. MOSFIRE was developed by teams from the UCLA, the Caltech, and the UCSC. The CoPrincipal Investigators were Dr. Ian S. McLean (UCLA) and Dr. Charles C. Steidel (Caltech), and the optical design was led by Harland Epps (UCSC). MOSFIRE was delivered to WMKO on February 8, 2012 and "first light" on the Keck 1 telescope was obtained on April 4, 2012. The performance of the detector and the as-built instrument, based on early commissioning results was presented in Kulas, McLean, and Steidel (2012) $)^{31}$ and McLean et al. (2012) ${ }^{32}$.
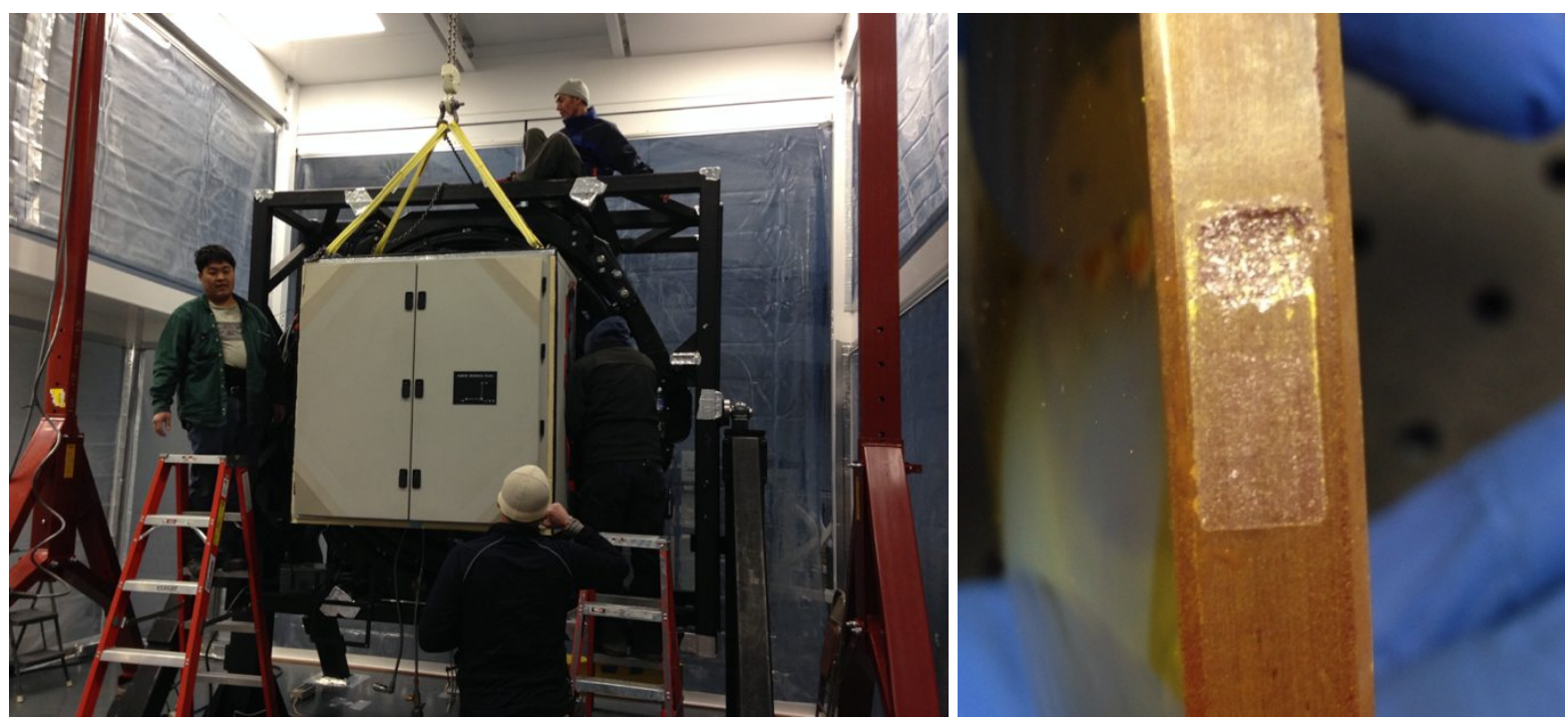

Figure 13: MOSIFE in the recently assembled clean room with the repair team working to remove the electronics cabinet from the back of the instrument to gain access to the dewar rear cover (left). The ZnSe collimator lens is viewed edge on (right) and shows one of six locations along the edge of the lens where the bond failed. This bond location shows evidence where the bond pulled out a chip from the lens, but four of six locations separated without chipping.

In late 2016, MOSFIRE was taken off-sky for six months to complete a repair of the optical system that resulted from rotator testing and tuning. Because WMKO did not have a clean environment large enough to accommodate MOSFIRE, a clean room was assembled in the Keck I dome. The clean room was outfitted with a portable 2-ton crane, the dome floor was reinforced, and an instrument stand was installed that enabled manual tilts of the instrument to facilitate 
removal of external and internal dewar components. Members of the original MOSFIRE development team were significant allies in fixing MOSFIRE. Both Co-PIs were present when opening the instrument and together with instrument technicians Hector Rodriguez from Caltech and Ken Mangnone from UCLA, the team provided guidance and training for observatory staff who had not seen MOSFIRE opened. This work delayed some projects at the observatory and because members of the MOSFIRE development team helped with the repair mission, projects at UCLA and Caltech were also delayed.

The repair team discovered that the second lens in the collimator barrel had come loose from six bonds. The collimator barrel was disassembled, and small particulates of lens debris was found in all layers of the collimator barrel. The damaged lens was sent back to UCLA along with the lens mount for rebonding by Ted Alido (UCLA) and Jason Fucik (Caltech). Although the lens coating had been partially damaged by rubbing against a neighboring lens, the lens remained in good enough quality to be reused. We reinstalled this lens rather than manufacture and recoat a new lens because the instrument down time would increase by several more months. After reinstalling the lens, MOSFIRE was returned to routine observing with no noticeable degradation in performance.

This event prompted the observatory to initiate new protocols and procedures when testing hardware or software that may have a potential to move facility instruments. But although the lens was loosened during rotator testing, the forces involved did not exceed forces encountered during normal installation of MOSFIRE at the Cassegrain focus. We suspect that the bonds of the lens were likely weaker that others due to lens preparation that differed from all other lenses in the system.

\section{ACKNOWLEDGEMENTS}

The data presented herein were obtained at the W. M. Keck Observatory, which is operated as a scientific partnership among the California Institute of Technology, the University of California, and the National Aeronautics and Space Administration. The Observatory was made possible by the generous financial support of the W. M. Keck Foundation. We wish to recognize and acknowledge the very significant cultural role and reverence that the summit of Maunakea has always had within the indigenous Hawaiian community. We are most fortunate to have the opportunity to conduct observations from this mountain.

For the projects described in this paper, there are several sources of funding we wish to acknowledge. The development of the blue channel of KCWI is supported in part by a grant from National Science Foundation under Scientific Program Order No. 5 as issued for support of the Telescope Systems Instrumentation Program (TSIP), in accordance with Proposal No. AST-0335461 submitted by AURA. Support for KCWI has also come from the Heising-Simons Foundation and the Mount Cuba Foundation. KCRM is supported in part by the National Science Foundation under Grant No. AST-1429890. The OSIRIS spectrograph upgrade including DRP development is supported by the National Science Foundation under Grant No. AST-1311102 while the imager upgrade project is supported by funding from the Gordon and Betty Moore Foundation. K1DM3 is supported in part by the National Science Foundation under Grant No. AST-1337609. The NIRSPEC upgrade is supported in part by the National Science Foundation under Grant No. AST1532315 and by the Heising-Simons Foundation. The wave front controller supported by the National Science Foundation under Grant AST-1727071. Development of KPF is supported by the Heising-Simons Foundation.

\section{REFERENCES}

[1] A.Kinney, S.Kulkarni, C.Max, H. Lewis, J.Cohen, C. L.Martin, C. Beichman, D.R.Ciardi, E.Kirby, J.Rhodes, A. Shapley, C. Steidel, S. Wright, R. Campbell, “The W. M. Keck Observatory Scientific Strategic Plan” WMKO July 2016 https://www2.keck.hawaii.edu/inst/newsletters/Vol20/scientificstrategicplan.pdf (July 2016)

[2] M. Matuszewski, C. Martin, P. Morrissey, "KCWI: a flexible integral field spectrograph at WM Keck Observatory," Proc SPIE, 10702-142 (2018)

[3] P. Morrissey, C. Martin, M. Matuszewski, D. Neill, Luca Rizzi, A. M. Moore, "The Keck Cosmic Web Imager: first light," Proc SPIE, 10702-2, (2018)

[4] L. Rizzi "KCWI Home Page," WMKO January 2017 https://www2.keck.hawaii.edu/inst/kcwi/ (August 2017)

[5] S. H. Kwok, K. Tsubota, T. Krasuski, J. Lyke, B. McCarney, J. Mader, K. McCann "Keck Observatory telescope control system upgrade status report," Proc SPIE, 10704-25, (2018)

[6] T Krasuski, P. M. Thompson, B. McCarney, S. Kwok, K. Tsubota, E. James, M. Pollard, K. McCann "Implementation of W. M. Keck Observatory's telescope control system upgrade," Proc SPIE, 10700-202 (2018) 
[7] Boehle, A., Larkin, J. E., Adkins, S. M. Aliado, T., Fitzgerald, M. P., Johnson, C. A., Lyke, J. E., Magnone, K. G., Sohn, J. M., Wang, E., and Weiss, J. L., "Upgrade of the detector in the integral field spectrograph OSIRIS at the W. M. Keck Observatory," Proc. SPIE, 9908, 104 (2016)

[8] Rizzi, L. Lyke, J. E. "Keck Observatory DRPs" WMKO January 2016 https://github.com/KeckDataReductionPipelines/OsirisDRP (2017)

[9] P. Arriaga, M. P. Fitzgerald, C. Johnson, J. L. Weiss, J. Canfield, T. Aliado, K. Magnone, J. M. Sohn, J. E. Lyke, A. Ghez, "Detection performance of the upgraded OSIRIS imager," Proc. SPIE, 10702-103 (2018)

[10] S. Ragland, P. L. Wizinowich, J. R. Lu, L. Jolissaint, G. B. Berriman, W. Best, T. J. Dupuy, C. R. Gelino, M. C. Liu, J. A. Mader, M. A. Van Dam, A. Vayner, G. Witzel, S. A. Wright, "Status of point spread function determination for Keck adaptive optics," Proc. SPIE, 10703-59 (2018)

[11] G. Witzel, J. R. Lu, A. Ciurlo, T. Do, M. P. Fitzgerald, A. M. Ghez, R. Campbell, O. Beltramo-Martin , C. M. Correia, S. Ragland, "PSF reconstruction for NIRC2/Keck: application of AIROPA to Galactic Center observations," Proc. SPIE, 10703-92 (2018)

[12] J. R. Lu, M. R. Chun, F. Abdurrahman, O. Lai, M. Service, D. Toomey, Dora Fohring, M. Kassis, S. M. Ammons, C. Martins, P. L. Wizinowich, R. G. Dekany, D. Gavel, C. Baranec, T. Do, L. Wang, C. Steideil, , "PSF reconstruction for NIRC2/Keck: application of AIROPA to Galactic Center observations," Proc. SPIE, 10703-23 (2018)

[13] P. L. Wizinowich, M. Chun, D., Mawet, G. Agapito, R. Dekany, S. Esposito, F. Thierry, O. Guyon, D. Hall, C. Plantet, R. Francois, "Near-infrared wavefront sensing," Proc. SPIE, 9909, 13 (2016)

[14] Femenia-Castella, B., Wizinowich, P., Rampy, R., Cetre, S., Lilley, S., Lyke, J., Ragland, S., Stomski, P., van Dam, M., "Status and new developments with the Keck I near-infrared tip-tilt sensor," Proc SPIE 990915 (2016)

[15] Ratliff, C. T., Cabak, G., Phillips, A., Prochaska, X., Deich, W., Sandford, D., Nelson, J., Cowley, D., Tripsas, A., Bolte, M., Park, S., Peck, M., Zachary, P., Wold, T., Dahler, M., Kassis, M., Vandenberg, A., Ward, J., "Fabrication completion and commissioning of a deployable tertiary mirror for the Keck I Telescope" Proc. SPIE, 10706-34, (2018)

[16] McLean, I. S., Becklin, E. E., Bendiksen, O., Brims, G., Canfield, J., Figer, D. F., Graham, J. R., Hare, J., Lacayanga, F., Larkin, J. E., Larson, S. B., Levenson, N., Magnone, N., Teplitz, H., and Wong, W., "Design and development of NIRSPEC: a near-infrared echelle spectrograph for the Keck II telescope", Proc. SPIE 3354, 566578 (1998).

[17] Martin, E. E. , Fitzgerald, M. P., McLean, I. S., Doppmann, G., Kassis, M., Canfield, J., Johnson, C., Kress, E., Lanclos, K., Magnone, K., Sohn, J. M., Wang, E., Weiss, J., "An overview of the NIRSPEC upgrade for the Keck II telescope," Proc SPIE, 10702-9, (2018)

[18] Gibson, S. R., Howard, A., Roy, a., Smith, C., Halverson, S., Edelstein, J., Kassis, M., Wishnow, E. H. Raffanti, M. P., Allen, S., Chin, J., Coutts, D., Cowley, D., Curtis, J., Deich, W., Feger, T., Finstad, D., Gurevich, Y., Ishikawa, Y., James, E., Jhoti, E., Lanclos, K., Lilley, S., Miller, T., Milner, S., Payne, T., Rider, K., Rockosi, C., Sandford, D., Schwab, C., Seifahrt, A., Sirk, M., Smith, R., Stuermer, J., Weisfeiler, M., Wilcox, M., Vandenberg, A., "Keck Planet Finder: Preliminary Design," Proc SPIE, 10702-216 (2018)

[19] S. Cetre, O. Guyon, C. Z. Bond, M. R. Chun, S. B. Goebel, C. Lockhart, D. Mawet, E. Wetherell, P. L. Wizinowich "A near-infrared pyramid wavefront sensor for Keck adaptive optics: real-time controller" Proc. SPIE, 10703-119 (2018)

[20] S. J. Lilley, P. L. Wizinowich, A. Vandenberg, M. Taheri, J. K. Wallace, M. R. Chun, C. Z. Bond, S. M. Jacobson, "A near-infrared pyramid wavefront sensor for Keck adaptive optics: opto-mechanical", Proc. SPIE, 10703-127 (2018)

[21] C. Z. Bond, S. Cetre, M. R. Chun, B. Femenia-Castella, D. N. B. Hall, S. B. Goebel, S. M. Jacobson, S. J. Lilley, C. Lockhart, E. A. Warmbier, D. Mawet, N. Jovanovic, O. Guyon, C. Plantet, G. Agapito, C. Giordano, S. Esposito, J. K. Wallace, E. Wetherell, P. L. Wizinowich, “Adaptive optics with an infrared pyramid wavefront sensor," Proc. SPIE, 10703-72 (2018)

[22] J. Delorme, D. Mawet, N. Jovanovic, J. K. Wallace, R. D. Bartos, P Wizinowich, G Ruane, "A fiber injection unit for Keck: final design and first results," Proc. SPIE, 10702-77 (2018)

[23] Lu, J. R., Chun, M., Abdurrahman, F., Lai, O., Service, M., Toomey, D., Fohring, D., Kassis, M., Ammons, S. M., Martins, C., Wizinowich, P., Dekany, R., Gavel, D., Baranec, C., Do, T., Wang, L., Steideil, C., "Science metrics and image quality for ground layer adaptive optics systems" Proc SPIE, 10703-23, (2018) 
[24] Chun, M. R., Lu, J. R., Lai, O., Abdurrahman, F., Service, M., Toomey, D., Fohring, D., Baranec, C., Hayano, Y., Oya, S., "On-sky results from the wide-field ground-layer adaptive optics demonstrator 'imaka," Proc. SPIE, 10703$19(2018)$

[25]Bundy, K., Savage, M., Kupke, R., MacDonald, N., Westfall, K., Radovan, M., Cai, Z., Digiorgio, B, Dekany, R., Divakar, D., Fucik, J., Ji, H., Miyazaki, S., Ozaki, S., Phillips, A., Roy, N., Smith, R., Surya, A., Nadar, S. P., Thirupathi, S., Tsuzuki, T., "WFOS instrument trade study: slicer vs. fiber instrument concept designs and results," Proc. SPIE, 10702-72 (2018)

[26] Kupke, R., Ji, H., Nadar, S. P. , Divakar, D., Gilles, L., "The wide field optical spectrograph (WFOS) for TMT: fiber-WFOS optical design," Proc. SPIE 10702-73 (2018)

[27] Martini, P., Besuner, R, Flaugher, B., Levi, M., Rockosi, C., Schlegel, D. J., Sprayberry, D., Bailey, S., Doel, P., Edelstein, J., Honscheid, K., Eisenstein, D., Wechsler, R., Gutierrez, G., Harris, S., Poppett, C., Silber, J. H., Joyce, R., "Overview of the Dark Energy Spectroscopic Instrument," Proc. SPIE, 10702-51, (2018)

[28] Mazin, B., "KRAKENS: a general purpose MKID integral field spectrograph for the Keck I telescope," Proc. SPIE, 10702-16 (2018)

[29] Larkin, J. E., Wright, S. A., Chisholm, E. M., Andersen, D., Dekany, R. G., Dunn, J. S., Ellerbroek, B. L., Hayano, Y., Kupke, R., Moore, A. M., Phillips, A. C., Simard, L., Smith, R. M., Suzuki, R., Trapp, A., Walth, G., Weber, R., Wincentsen, J. E., Weiss, J. L., Zhang, K., "The infrared imaging spectrograph (IRIS) for TMT: instrument overview," Proc. SPIE, 10702-65, (2018)

[30] Skemer, A. J., Stelter, D., Mawet, D., Marois, C., Fitzgerald, M., Mazin, B., Guyon, O., Brandt, T., Chilcote, J., Delorme, J., Jovanovic, N., Lu, J., Millar-Blanchaer, M., Wallace, K., Vasisht, G., Roberts, L., Wang, J., “The Planetary Systems Imager: 2-5 Micron Channel," Proc. SPIE, 10702-371, (2018)

[31] Kulas, K. R., McLean, I. S. and Steidel, C. C., "Performance of the HgCdTe detector for MOSFIRE, an imager and multi-object spectrometer for Keck Observatory," Proc. SPIE 8453, 84531S, 10 pp. (2012).

[32] McLean, I. S., Steidel, C. C., Epps, H. W., Konidaris, N., Matthews, K. Y., Adkins, S., Aliado, T., Brims, G., Canfield, J. M., Cromer, J. L., and 10 coauthors, "MOSFIRE, the multi-object spectrometer for infrared-red exploration at the Keck Observatory," Proc. SPIE 8446, 844600J, 15 pp. (2012). 